\title{
Seeing and the Ability to See: A Framework for Viewing Geometric Cube Problems
}

\author{
Kok Xiao-Feng Kenan ${ }^{1 *}$ \\ ${ }^{1}$ St. Margaret's Secondary School, SINGAPORE \\ *CORRESPONDENCE: 《kenankok@gmail.com

\begin{abstract}
Perceiving a 3-dimensional (3D) diagram on a 2-dimensional (2D) surface or plane can be a challenging endeavor for students at the elementary or primary grade levels. Adding to this challenge are the intricacies present in understanding the processes involved in geometric problems of such a nature. To ease the comprehension of these processes, this paper proposes a framework that traces the processes in viewing $3 \mathrm{D}$ diagrams represented on a $2 \mathrm{D}$ plane. This framework, abbreviated as SMS, espouses three main processes; (1) Seeing the 2D plane, (2) Making sense of the 3D diagram on the 2D plane, and (3) Seeing the 3D diagram. Implications for teaching and learning are also offered.
\end{abstract}

Keywords: cube, geometry, 3-dimensional, 2-dimensional, visual perception, visualisation, spatial visualisation

\section{INTRODUCTION}

Perceiving a 3-dimensional (3D) diagram on a 2-dimensional (2D) surface or plane can be a challenging endeavour for students at the elementary or primary grade levels. Research has extensively documented the difficulties that students face with regards to problems of such a nature. It was reported that less than $50 \%$ of middle-grade students (Ben-Haim et al., 1985) could solve problems involving seeing a 3D diagram represented on a $2 \mathrm{D}$ surface (see Figure 1). In addition, less than $40 \%$ of 17 year old students were found to be able solve such problems as enumerated by the National Assessment of Educational Progress (NAEP) results (Hirstein, 1981). Both these sets of statistics highlight that there is a fundamental difficulty associated with such problems. But what exactly are these difficulties?

These difficulties are best summarized by Ben-Haim et al. (1985) who divided the errors grades 5 to 8 students made into four categories based on the 3D representation in Figure 1. The first and second categories are counting the number of cube faces as directly seen from the diagram and doubling that number respectively. The third category is counting the number of cubes in the diagram while the fourth category is counting the number of cubes in the diagram and doubling that number.

However, the processes that can be attributed to these difficulties remain to be foregrounded. It was suggested that the first category of error was due to perceiving the diagram simply as a $2 \mathrm{D}$ object. At this point, it is necessary to ask, what process is involved in causing the students to perceive the diagram as a $2 \mathrm{D}$ object and not as a 3D object? This question cannot be answered without understanding the processes present in viewing problems of this nature.

Another point of interest is the absence of a doubling of the count, which alludes to the first and third categories of errors. It was suggested this was due to the inability to visualize the unseen portions of the

Article History: Received 22 April $2018 \bullet$ Revised 6 May $2018 \bullet$ Accepted 26 May 2018

(C) 2018 The Author(s). Open Access terms of the Creative Commons Attribution 4.0 International License (http://creativecommons.org/licenses/by/4.0/) apply. The license permits unrestricted use, distribution, and reproduction in any medium, on the condition that users give exact credit to the original author(s) and the source, provide a link to the Creative Commons license, and indicate if they made any changes. 
How many unit cubes

does it take to make this rectangular solid?

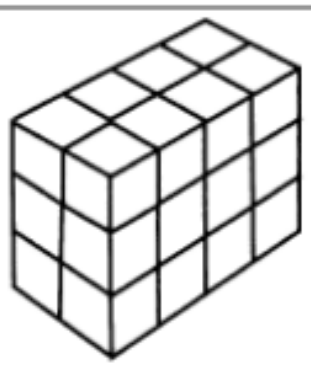

[Source: Battista, M. T. \& Clements, D. H. (1996). Students' Understanding of Three-Dimensional Rectangular Arrays of Cubes. Journal for Research in Mathematics Education, 27(3), 259.]

Figure 1. Typical Geometric Cube Problem

diagram. However, what process constitutes not being able to visualize these hidden portions? Again, an understanding of the processes behind such problems will go a long way in addressing this question.

What is needed, therefore, is a framework that outlines the processes in viewing 3D diagrams represented on $2 \mathrm{D}$ planes so that the difficulties experienced by students can be accurately pinpointed to the process involved. Strategies can then be developed to address the difficulties associated with the identified process.

\section{PURPOSE}

The aim of this paper therefore, is to provide a framework that traces the processes involved in viewing 3dimensional (3D) diagrams represented on a 2-dimensional (2D) plane. The framework will outline three main processes in viewing such geometric problems as gathered from the literature.

\section{SIGNIFICANCE}

This framework is important in a number of ways. Firstly, it enables teachers to understand the intricacies of the processes that pertain to such geometric cube problems. This would put teachers in a better position to assess the difficulties their students have and directly pinpoint the processes where the difficulties lay. Such is advantageous for the diagnosis of errors and probing of students' thinking into viewing $3 \mathrm{D}$ diagrams represented on a 2D surface. Secondly, this framework could pave the way for how mental images are formed as it provides insights into each process of viewing such problems.

\section{FRAMEWORK}

There are three main processes in this proposed framework which will be abbreviated as SMS. They are as follows:

1. Seeing the $2 \mathrm{D}$ plane,

2. Making sense of the 3D diagram on the $2 \mathrm{D}$ plane, and

3. Seeing the 3D diagram.

The process of seeing the 2D plane is the first stage of approaching geometric problems involving viewing $3 \mathrm{D}$ diagrams represented on a $2 \mathrm{D}$ plane. At this stage, vision or visual perception is required to gain direct access to the 2D plane where the 3D diagram is represented on. According to Duval (1999), this is epistemological function of visual perception. In addition, visual perception allows the entire field of the $2 \mathrm{D}$ plane where the 3D diagram is represented on to be comprehended, which is the synoptic function of visual perception (Duval, 1999).

However, one important question needs to be addressed. What exactly is comprehended in this 2D field? Herein lays the limitation of visually perceiving. As we live in a 3D world, we can only see the current view of the $2 \mathrm{D}$ plane as it stands. To completely perceive the $3 \mathrm{D}$ diagram represented in the $2 \mathrm{D}$ plane, we need to either change our view of looking at the diagram from different angles or mentally rotate the diagram to see the other sides. It must be acknowledged however, that changing our view of looking at the diagram from 
different angles would be extremely difficult unless the diagram is first perceived as a 3D object on a $2 \mathrm{D}$ plane. This was a difficulty that Ben-Haim et al. (1985) found in a study involving grades 5 to 8 students as mentioned above. It was postulated that students saw the diagram as a $2 \mathrm{D}$ diagram represented on a $2 \mathrm{D}$ plane rather than a $3 \mathrm{D}$ diagram represented on a $2 \mathrm{D}$ plane, hence causing the students to count the number of cube faces on the diagram without taking into account the cubes not directly accessible visually.

Therefore, to comprehend the $3 \mathrm{D}$ diagram represented on the $2 \mathrm{D}$ plane, one needs to see that the $3 \mathrm{D}$ diagram is indeed 3D. How should one see that it is a 3D diagram represented on a $2 \mathrm{D}$ plane in the first place? This is where one must understand that the 2D plane is only used to represent the 3D diagram and does not therefore provide a direct apprehension to the 3D diagram. To directly apprehend the 3D diagram, one needs to make meaning and sense of the representation first. This would involve the ability to visualise, which is the second process in this proposed framework.

Making sense of the 3D diagram on the 2D plane requires the ability of visualisation. Visualisation at its core is essentially about making meaning and sense (Zaskis, Dubinsky and Dauterman, 1996; Duval, 1999) of whatever is seen. In this case, it is taking the representation of the $3 \mathrm{D}$ diagram and making sense and meaning of it as a 3D diagram. It is the action of creating a strong relationship between a mental construction of the represented 3D diagram and the 2D plane through which the 3D diagram is represented on (Zaskis, Dubinsky \& Dauterman, 1996). This connection is possible through constructing the represented 3D diagram mentally after associating with its presentation in the $2 \mathrm{D}$ plane.

However, even if one can make sense and mentally construct the represented 3D diagram, it may not guarantee a complete apprehension of the 3D diagram. This is because the 3D diagram needs to be viewed from different angles in order that it be fully understood. Hence, this alludes to the third process of seeing the $3 \mathrm{D}$ diagram in its totality.

Seeing the $3 \mathrm{D}$ diagram in its totality necessitates mentally rotating the diagram and not just mentally constructing the diagram. This demands the ability to spatially visualise, which is the ability to see depth in a diagram. In particular, the mental manipulation of the represented 3D diagram is required (McGee, 1979; NCTM, 2000) to see the different perspectives such as the top, side and front views. This could entail mentally rotating, twisting or even inverting a represented 3D diagram (McGee, 1979).

Therefore, it is evident that the three processes, as mentioned above, are essential for viewing $3 \mathrm{D}$ diagrams represented on $2 \mathrm{D}$ planes. These three processes should be regarded as inseparable and interconnected when approaching problems of such a nature. I contend that the three processes should be seen in totality and in relation to one another. The limitations of visual perception can be addressed by the ability to visualise, while spatial visualisation fills the void left by visualisation.

Figure 2 illustrates the flow of the three processes when viewing $3 \mathrm{D}$ diagrams represented on a $2 \mathrm{D}$ plane.

\section{Seeing the 2D plane}

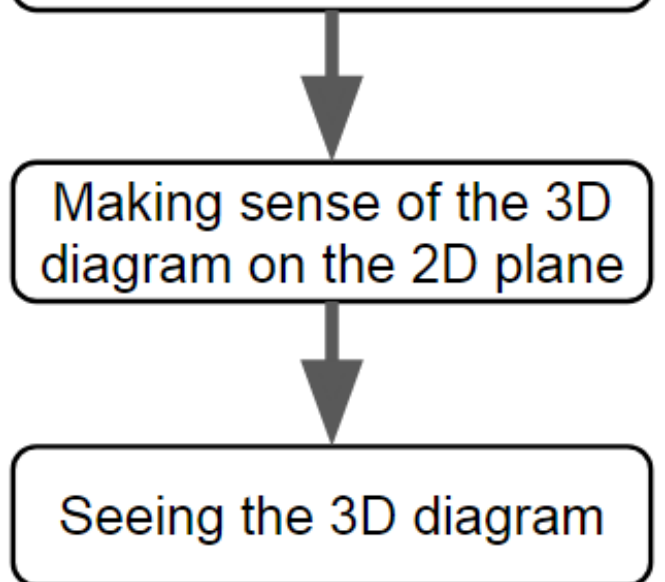

Figure 2. The SMS Framework for Viewing 3D Diagrams Represented on a 2D Plane 


\section{IMPLICATIONS FOR TEACHING \& LEARNING}

This framework has the potential to be a tool for the diagnosis of errors in students' thinking when approaching such problems requiring the viewing of a represented 3D diagram on a $2 \mathrm{D}$ surface. Activities can then be designed to address these faults in thinking. For instance, students who make the error of counting the cube surfaces in Figure 1 would likely have issues with seeing the 2D diagram. They therefore have problems comprehending the 3D diagram on the 2D plane and making sense of it. Thus, it would be necessary to address two issues. Firstly, the visual perception of students which refers to what students actually see. Secondly, their ability to visualise needs to be addressed which is about how students see represented 3D diagrams on a $2 \mathrm{D}$ plane.

In addition, teachers could use this framework to craft activities to target specific processes that students have difficulties with. This would enhance the students' learning as it addresses the root of their error or difficulty. Teachers could even use this framework to structure their lessons in geometry topics involving viewing $3 \mathrm{D}$ diagrams represented on $2 \mathrm{D}$ surfaces, such as $3 \mathrm{D}$ trigonometry. It is important to gradually and methodologically build up students' competency and ability in visualisation and spatial visualisation.

\section{CONCLUSION}

The proposed SMS framework has provided a way to view the processes involved in viewing 3D diagrams represented on $2 \mathrm{D}$ surfaces. This, I believe, provides not only a way to understand the processes present in viewing such problems, but also functions as a tool for teachers to diagnose difficulties that their students experience with such geometric problems. Therefore, I advocate the use of such a framework to guide teachers in comprehending geometric problems of this nature.

\section{Disclosure statement}

No potential conflict of interest was reported by the authors.

\section{Notes on contributors}

Kok Xiao-Feng Kenan - Teacher, St. Margaret's Secondary School, Singapore.

\section{REFERENCES}

Battista, M. T., \& Clements, D. H. (1996). Students' Understanding of Three-Dimensional Rectangular Arrays of Cubes. Journal for Research in Mathematics Education, 27(3), 258-292.

Ben-Haim, D., Lappan, G., \& Houang, R. T. (1985). Visualizing Rectangular Solids Made of Small Cubes: Analyzing and Effecting Students' Performance. Educational Studies in Mathematics, 16(4), 389-409. https://doi.org/10.1007/BF00417194

Duval, R. (1999). Representation, vision and visualization: Cognitive functions in mathematical thinking. Basic issues for learning. In F. Hitt \& M. Santos (Eds.), Proceedings of the 21st North American PME Conference, 1, 3-26.

Hirstein, J. J. (1981). The second national assessment in mathematics: Area and volume. The Mathematics Teacher, 74(9), 704-708.

McGee, M. G. (1979). Human spatial abilities: Sources of sex differences. New York: Praeger.

National Council of Teachers of Mathematics. (2000). Principles and Standards for School Mathematics. Reston, Va.: NCTM, 2000.

Zazkis, R., Dubinsky, E., \& Dauterman, E. (1996). Using visual and analytic strategies: A study of students' understanding of permutation and symmetry groups. Journal for Research in Mathematics Education, 27, 435-475. https://doi.org/10.2307/749876 